\title{
Patient-reported bowel and bladder function is not adversely impacted by bariatric surgery
}

\author{
Michelle Campbell ${ }^{1,2}$ - Eliza A. Conaty ${ }^{3} \cdot$ Mikhail Attaar $^{1,2} \cdot$ Hoover Wu $^{1,2} \cdot$ Harry J. Wong $^{1,2} \cdot$ Kristine Kuchta $^{4}$.

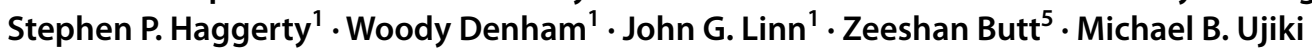

Received: 3 September 2021 / Accepted: 3 January 2022 / Published online: 7 February 2022

(c) The Author(s), under exclusive licence to Springer Science+Business Media, LLC, part of Springer Nature 2022

\begin{abstract}
Introduction This study aims to assess the effect of bariatric surgery on patient-reported outcomes of bowel and bladder function. We hypothesized that bariatric surgery does not worsen bowel and bladder function.

Methods and procedures A retrospective review was conducted of a prospectively maintained surgical quality database. We included patients who underwent primary bariatric surgery at a single institution between 2012 and 2020, excluding revisional procedures. Patient-reported outcomes were assessed using Surgical Outcomes Measurement System (SOMS) bowel and bladder function questionnaires at time of pre-operative consult and routine post-operative follow-up visits through 2 years. Data were analyzed using a statistical mixed effects model.

Results 573 patients ( $80.6 \%$ female) were identified with completed SOMS questionnaire data on bowel and bladder function. Of these, 370 (64.6\%) underwent gastric bypass, 190 (33.2\%) underwent sleeve gastrectomy, and 13 (2.3\%) underwent either gastric banding or duodenal switch. Compared to pre-operative baseline scores, patients reported a transient worsening of bowel function at 2-weeks post-op $(p=0.009)$. However, by 3-months post-op, bowel function improved and was significantly better than baseline ( $p=0.006)$; this improvement was sustained at every point through 2-year follow-up $(p=0.026)$. Bladder function scores improved immediately at 2-weeks post-op $(p=0.026)$ and showed sustained improvement through 1-year follow-up. On subgroup analysis, sleeve patients showed greater improvement in bowel function than bypass patients at 1-year $(p=0.031)$. Multivariable analysis showed significant improvement in bowel function associated with greater total body weight loss (TBWL) $(p=0.002)$.

Conclusions Bariatric surgery does not worsen patient-reported bowel or bladder function. In fact, there is overall improvement from pre-operative scores for both bowel and bladder function by 3-months post-op which is sustained through 2-year and 1-year follow-up, respectively. Most encouragingly, a greater TBWL is significantly associated with improved bowel function after bariatric surgery.
\end{abstract}

Keywords Bariatric surgery $\cdot$ Bowel function $\cdot$ Bladder function $\cdot$ Quality of life $\cdot$ SOMS

Michelle Campbell

Michelle.Campbell2@uchospitals.edu

1 Department of Surgery, NorthShore University HealthSystem, 2650 Ridge Ave, GCSI Suite B665, Evanston, IL 60201, USA

2 Department of Surgery, University of Chicago Medical Center, Chicago, IL, USA

3 Stritch School of Medicine, Loyola University Chicago, Chicago, IL, USA

4 NorthShore University Research Institute, Evanston, IL, USA

5 Feinberg School of Medicine, Northwestern University, Chicago, IL, USA
Obesity is a global public health crisis associated with significant comorbidities and negative impacts on both quality of life and overall life expectancy. In 2014, the prevalence of obesity in the United States was $35.0 \%$ amongst men and $40.4 \%$ amongst women [1]. Weight loss procedures and interventions have been evolving over the last 60 years, with Roux-en-Y gastric bypass (RYGB) and sleeve gastrectomy rising to predominance in the last two decades [2]. Bariatric surgery offers the most effective means of durable weight loss, and evidence demonstrates significant improvement in obesity-related comorbidities such as diabetes mellitus, hypertension, and dyslipidemia following bariatric surgery [3-6]. However, bariatric surgery carries a reputation for 
causing worsening of gastrointestinal (GI) symptoms such as flatulence, fecal urgency, diarrhea, and abdominal cramping. Fear of these symptoms may contribute to the underutilization of bariatric surgery in patients with morbid obese who otherwise qualify for intervention.

The existing evidence is mixed on the effects of bariatric surgery on GI symptoms. Malabsorptive procedures such as RYGB and duodenal switch have been associated with postoperative loose stools or diarrhea, malodorous flatulence, and dumping syndrome [7-10]. However, other studies demonstrate an improvement in GI symptoms after RYGB, including improved symptoms of reflux, indigestion, abdominal pain, and fecal incontinence [11-14]. Studies are limited by non-standardized symptom questionnaires and small sample sizes, resulting in low-quality evidence. There have been reports of improved urinary incontinence following bariatric surgery [15], however, relatively little is known about the effect on patient experience with urinary symptoms following bariatric surgery.

In the current study, we aim to assess the effect of bariatric surgery on patient-reported outcomes of bowel and bladder function using a previously validated symptom questionnaire tailored for use in the post-surgical population. We hypothesized that bariatric surgery does not worsen bladder or bowel function.

\section{Methods}

\section{Data collection}

We performed a retrospective review of a single institution surgical quality database. Preoperative, intraoperative, and postoperative data are collected prospectively through review of the electronic medical record. Patients who underwent primary bariatric surgery, including sleeve gastrectomy (SG), Roux-en-Y gastric bypass (RYGB), duodenal switch (DS), and adjustable gastric banding (AGB) between January 2012 and June 2021, and had available quality of life questionnaire responses were included. Revisional procedures were excluded. Internal review board approval was obtained for this study.

\section{Perioperative protocol}

All patients undergo a comprehensive multidisciplinary bariatric evaluation prior to their operation. Preoperative and postoperative care follows clinical practice guidelines published by the American Society for Metabolic and Bariatric Surgery (ASMBS)[16]. All patients are required to undergo preoperative esophagogastroduodenoscopy (EGD) and laboratory testing, including comprehensive metabolic panel (CMP), complete blood count (CBC), glycosylated hemoglobin (HbA1c), and vitamin levels. Additionally, patients are required to cease smoking and are placed on a liquid diet for two to six weeks prior to the day of surgery; duration of liquid diet is dependent on consult BMI. Surgical procedure is determined at the discretion of the operating surgeon following a discussion with the patient of individual patient factors such as body mass index (BMI), comorbidity status, functional status, and patient preference.

Postoperatively, patients are admitted until they demonstrate adequate pain control utilizing oral narcotics, urinate without catheter assistance, and tolerate a liquid diet. Patients are instructed to adhere to a full liquid diet, avoiding caffeinated, carbonated, and alcoholic beverages, for two weeks. After postoperative day 14, patients advance to pureed and soft diets for an additional two weeks. Vitamin and mineral supplementation are strongly encouraged, as well as ongoing follow-up with registered dietitians.

\section{Surgical outcomes measurement system}

Patient-reported quality of life data are routinely collected on all bariatric patients at this institution using the Surgical Outcomes Measurement System (SOMS) questionnaire, designed specifically for use in the postoperative population. Questionnaire domains are tailored to the bariatric population and are administered to all patients preoperatively and postoperatively at all routine follow-up visits (two weeks, six weeks, three months, six months, and annually). This study reports exclusively on the bowel function and bladder function domains, which include questions addressing symptom frequency within the seven days prior. Bowel function includes four questions with a total possible score 4-20; higher score indicates worse function. Bladder function includes three questions with a total possible score 30-15; higher score indicates worse function. Questionnaires were administered during in-person clinic visits until July 2020, at which time questionnaires were distributed through online access due to the use of telehealth during the COVID-19 pandemic.

\section{Statistical analysis}

Patient and clinical characteristics were summarized using mean with standard deviation, median with interquartile range, or frequency with percentage. Comparisons of postoperative SOMS scores to baseline were made using the paired t-test. Subgroup comparisons of SOMS scores between GB and SG patients were performed using the Wilcoxon rank-sum test. Linear mixed effects models with random intercepts for each patient were used to assess factors associated with bladder and bowel function. Fixed effects covariates in the models included timepoint, sex, age, surgery type and TBWL. All statistical tests were two-tailed 
Table 1 Patient Characteristics

\begin{tabular}{ll}
\hline Total patients $[\mathrm{N}]$ & 573 \\
\hline Sex $[N(\%)]$ & \\
Male & $111(19.4)$ \\
Female & $462(80.6)$ \\
Age at surgery, years [Mean $\pm \mathrm{SD}]$ & $47 \pm 12$ \\
Consult BMI, kg/m ${ }^{2}[\mathrm{Mean} \pm \mathrm{SD}]$ & $46.0 \pm 7.8$ \\
Preop BMI, kg/m² $[\mathrm{Mean} \pm \mathrm{SD}]$ & $43.5 \pm 6.8$ \\
Race $[N(\%)]$ & \\
White/Caucasian & $383(66.8)$ \\
Black/African American & $87(15.2)$ \\
Hispanic & $35(6.1)$ \\
Other/unknown & $68(11.9)$ \\
Smoker ${ }^{\mathrm{a}}[\mathrm{N}(\%)]$ & $29(5.1)$ \\
Diabetes $[N(\%)]$ & \\
No & $444(77.5)$ \\
Non-insulin dependent & $85(14.8)$ \\
Insulin dependent & $44(7.7)$ \\
Surgery Type $[N(\%)]$ & \\
Gastric bypass & $370(64.6)$ \\
Sleeve & $190(33.2)$ \\
Other (lap band, duodenal switch) & $13(2.3)$ \\
Operative time, minutes $[\mathrm{Mean} \pm \mathrm{SD}]$ & $90 \pm 33$ \\
Length of stay, days $[\mathrm{Median}(\mathrm{Q} 1-\mathrm{Q} 3)]$ & $2(1-2)$ \\
30-Day readmission $[\mathrm{N}(\%)]$ & $27(4.7)$ \\
30-Day reoperation $[\mathrm{N}(\%)]$ & $12(2.1)$ \\
\hline
\end{tabular}

$B M I$ body mass index

${ }^{\mathrm{a}}$ Within previous 12 months and performed using SAS v9.4 (SAS Institute, Cary, NC). Statistical significance was set at $\mathrm{p}<0.05$.

\section{Results}

\section{Patient and operative characteristics}

From January 2012 to June 2021, 573 patients (80.6\% female, $66.8 \%$ white) underwent primary bariatric surgery and had available SOMS questionnaire data. Procedures performed included gastric bypass $(N=370,64.6 \%)$ and sleeve gastrectomy $(N=190,33.2 \%)$ with gastric band or duodenal switch comprising the remaining $2.3 \%$. Average BMI at time of preoperative consult was $46.0 \pm 7.8$. Additional patient and operative characteristics are displayed in Table 1.

\section{Weight loss results}

One hundred twenty patients $(20.9 \%)$ had weight data available at one-year follow-up. One-year average total body weight loss (TBWL) was $74.4 \pm 27.7 \mathrm{lbs}$ for gastric bypass and $57.6 \pm 21.5 \mathrm{lbs}$ for sleeve, representing average percent excess weight loss (\%EWL) of $58.4 \pm 19.7 \%$ and $41.0 \pm 15.1 \%$, respectively. Weight loss results across all time points are summarized in Table 2 .

\section{Bowel function after bariatric surgery}

Patients reported an average 0.8-point increase from baseline in total bowel function score at two-week follow-up

Table 2 Weight loss results

\begin{tabular}{|c|c|c|c|c|c|c|c|c|}
\hline & $\begin{array}{l}\text { Consult } \\
\text { Mean } \pm \text { SD }\end{array}$ & $\begin{array}{l}\text { Preop } \\
\text { Mean } \pm \text { SD }\end{array}$ & $\begin{array}{l}2 \text { Weeks } \\
\text { Mean } \pm \text { SD }\end{array}$ & $\begin{array}{l}6 \text { Weeks } \\
\text { Mean } \pm \text { SD }\end{array}$ & $\begin{array}{l}3 \text { Months } \\
\text { Mean } \pm \text { SD }\end{array}$ & $\begin{array}{l}6 \text { Months } \\
\text { Mean } \pm \text { SD }\end{array}$ & $\begin{array}{l}1 \text { Year } \\
\text { Mean } \pm \text { SD }\end{array}$ & $\begin{array}{l}2 \text { Years } \\
\text { Mean } \pm \text { SD }\end{array}$ \\
\hline All patients $(N)$ & 128 & 363 & 222 & 33 & 46 & 135 & 120 & 73 \\
\hline Weight (lbs) & $282.6 \pm 59.4$ & $267.2 \pm 53.5$ & $254.4 \pm 48.9$ & $231.9 \pm 43.7$ & $224.4 \pm 45.9$ & $207.7 \pm 41.1$ & $199.6 \pm 47.7$ & $190 \pm 44.5$ \\
\hline BMI $\left(\mathrm{kg} / \mathrm{m}^{2}\right)$ & $46.0 \pm 7.8$ & $43.5 \pm 6.8$ & $41.5 \pm 6.4$ & $38.6 \pm 6.5$ & $36.8 \pm 6.2$ & $33.6 \pm 5.3$ & $32.4 \pm 6.0$ & $30.4 \pm 5.8$ \\
\hline TBWL (lbs) & - & $21.0 \pm 13.9$ & $9.6 \pm 5.2$ & $24.7 \pm 31.3$ & $34.5 \pm 11.8$ & $57.9 \pm 19.4$ & $69.6 \pm 28.2$ & $72.6 \pm 33.0$ \\
\hline$\%$ EWL (\%) & - & - & $7.7 \pm 4.0$ & $19.6 \pm 18.2$ & $29.4 \pm 11.0$ & $46.3 \pm 15.7$ & $53.7 \pm 20.8$ & $59.8 \pm 24.7$ \\
\hline Bypass (N) & 98 & 266 & 168 & 19 & 31 & 99 & 91 & 60 \\
\hline Weight (lbs) & $285.3 \pm 59.4$ & $266.7 \pm 52.0$ & $254.1 \pm 49.1$ & $227.9 \pm 40.5$ & $222.4 \pm 45.1$ & $204.8 \pm 40.9$ & $192.6 \pm 48.0$ & $187.2 \pm 45.5$ \\
\hline BMI $\left(\mathrm{kg} / \mathrm{m}^{2}\right)$ & $46.3 \pm 7.7$ & $43.7 \pm 6.8$ & $41.4 \pm 6.4$ & $38.2 \pm 5.9$ & $36.3 \pm 6.7$ & $33.2 \pm 5.1$ & $31.4 \pm 6.0$ & $29.8 \pm 5.5$ \\
\hline TBWL (lbs) & - & $21.4 \pm 13.3$ & $9.1 \pm 4.9$ & $20.7 \pm 6.4$ & $37.2 \pm 11.2$ & $60.4 \pm 18.4$ & $74.4 \pm 27.7$ & $76.5 \pm 29.4$ \\
\hline$\%$ EWL (\%) & - & - & $7.5 \pm 4.1$ & $19.0 \pm 7.3$ & $32.0 \pm 11.1$ & $48.6 \pm 14.4$ & $58.4 \pm 19.7$ & $63.3 \pm 22.0$ \\
\hline Sleeve $(N)$ & 26 & 90 & 53 & 10 & 10 & 31 & 23 & 9 \\
\hline Weight (lbs) & $271.6 \pm 61.8$ & $268.9 \pm 59.1$ & $254.7 \pm 49.1$ & $233.8 \pm 51.7$ & $225.1 \pm 52.1$ & $211.4 \pm 39.4$ & $218.7 \pm 40.4$ & $197.9 \pm 37.6$ \\
\hline BMI $\left(\mathrm{kg} / \mathrm{m}^{2}\right)$ & $45.3 \pm 8.6$ & $43.2 \pm 6.8$ & $42.0 \pm 6.5$ & $39.1 \pm 9.1$ & $37.7 \pm 5.7$ & $34.5 \pm 6.0$ & $35.3 \pm 5.0$ & $31.7 \pm 5.0$ \\
\hline TBWL (lbs) & - & $21.4 \pm 16.4$ & $11.3 \pm 5.7$ & $37.6 \pm 58.5$ & $32.9 \pm 9.9$ & $55.0 \pm 19.0$ & $57.6 \pm 21.5$ & $60.9 \pm 43.9$ \\
\hline \%EWL (\%) & - & - & $8.7 \pm 3.6$ & $24.2 \pm 33.2$ & $26.9 \pm 6.2$ & $42.8 \pm 16.9$ & $41.0 \pm 15.1$ & $48.0 \pm 28.1$ \\
\hline
\end{tabular}

$B M I$ body mass index, $T B W L$ total body weight loss, $E W L$ excess weight loss 
representing significant worsening in bowel function $(0.8 \pm 3.2, p=0.009)$. By three-month follow-up, total bowel function score had decreased to below preoperative baseline score, representing significant improvement in overall bowel function $(-1.1 \pm 2.7, p=0.006)$. Bowel function scores remained significantly improved from preoperative baseline through two-year postoperative follow-up $(-1.4 \pm 2.9$, $p=0.026$ ) for the entire cohort (Table 3 ). On comparison of gastric bypass versus sleeve patients (Fig. 1), there were no significant differences in scores except at one-year followup (gastric bypass $7.9 \pm 2.7$ vs sleeve $6.8 \pm 2.9, p=0.031$ ) at which time sleeve patients report significantly lower scores (better function) on the component question for urgent bowel movements (gastric bypass $1.8 \pm 0.9$ vs sleeve $1.5 \pm 0.8$, $p=0.048)$.

\section{Bladder function after bariatric surgery}

Patients reported an immediate significant improvement in bladder function two weeks postoperatively with an average decrease in symptom score of 0.4-points from preoperative baseline $(-0.4 \pm 1.7, p=0.026)$. Bladder function scores remained significantly improved from preoperative baseline through one-year postoperative follow-up $(-0.8 \pm 1.4, p=0.001)$ for the entire cohort (Table 3$)$. No significant differences were observed on subgroup analysis comparing bladder function scores in gastric bypass versus sleeve patients.

Table 3 SOMS bowel and bladder function scores

\begin{tabular}{|c|c|c|c|c|c|c|c|}
\hline & $\begin{array}{l}\text { Preop } \\
\text { Mean } \pm S D\end{array}$ & $\begin{array}{l}2 \text { Weeks } \\
\text { Mean } \pm \text { SD }\end{array}$ & $\begin{array}{l}6 \text { Weeks } \\
\text { Mean } \pm \text { SD }\end{array}$ & $\begin{array}{l}3 \text { Months } \\
\text { Mean } \pm \text { SD }\end{array}$ & $\begin{array}{l}6 \text { Months } \\
\text { Mean } \pm \text { SD }\end{array}$ & $\begin{array}{l}1 \text { Year } \\
\text { Mean } \pm \text { SD }\end{array}$ & $\begin{array}{l}2 \text { Years } \\
\text { Mean } \pm \text { SD }\end{array}$ \\
\hline All patients $(N)$ & 254 & 254 & 154 & 134 & 140 & 122 & 60 \\
\hline Total Bowel Function Score (4-20) & $7.9 \pm 2.5$ & $8.8 \pm 2.6$ & $8.1 \pm 2.4$ & $7.5 \pm 2.4$ & $7.1 \pm 2.5$ & $7.6 \pm 2.8$ & $7.2 \pm 2.5$ \\
\hline$p$-value from preop & - & 0.0088 & 0.6973 & 0.0059 & 0.0097 & 0.0384 & 0.0256 \\
\hline Total Bladder Function Score (3-15) & $4.5 \pm 1.4$ & $4.2 \pm 1.4$ & $3.9 \pm 1.4$ & $3.8 \pm 1.2$ & $3.9 \pm 1.2$ & $3.9 \pm 1.2$ & $4.0 \pm 1.2$ \\
\hline$p$-value from preop & - & 0.0257 & 0.0210 & 0.0004 & $<.0001$ & 0.0008 & 0.4254 \\
\hline Gastric bypass $(N)$ & 161 & 156 & 103 & 101 & 101 & 90 & 48 \\
\hline Urgent Bowel Movements (1-5) & $2.1 \pm 1.0$ & $1.8 \pm 0.9$ & $1.7 \pm 0.9$ & $1.6 \pm 0.7$ & $1.7 \pm 0.8$ & $1.8 \pm 0.9$ & $1.8 \pm 0.8$ \\
\hline Abdominal Cramping or Pain (1-5) & $1.9 \pm 0.9$ & $2.3 \pm 1.1$ & $1.9 \pm 0.9$ & $1.8 \pm 0.9$ & $1.7 \pm 0.8$ & $1.9 \pm 1.0$ & $1.7 \pm 0.8$ \\
\hline Loose or Liquid Stool (1-5) & $2.1 \pm 0.9$ & $2.3 \pm 1.1$ & $1.9 \pm 0.8$ & $1.7 \pm 0.7$ & $1.6 \pm 0.8$ & $1.9 \pm 0.9$ & $1.8 \pm 0.8$ \\
\hline Constipated (1-5) & $1.9 \pm 1.0$ & $2.5 \pm 1.2$ & $2.7 \pm 1.2$ & $2.5 \pm 1.1$ & $2.1 \pm 1.0$ & $2.2 \pm 1.1$ & $2.1 \pm 1.1$ \\
\hline Total Bowel Function Score (4-20) & $8.0 \pm 2.6$ & $8.9 \pm 2.7$ & $8.2 \pm 2.3$ & $7.6 \pm 2.4$ & $7.1 \pm 2.4$ & $7.9 \pm 2.7$ & $7.3 \pm 2.4$ \\
\hline$p$-value from preop & - & 0.0149 & 0.9524 & 0.0404 & 0.0055 & 0.0504 & 0.0322 \\
\hline Less than $2 \mathrm{~h}(1-5)$ & $2.3 \pm 1.2$ & $2.0 \pm 1.1$ & $1.7 \pm 1.0$ & $1.7 \pm 0.9$ & $1.8 \pm 1.1$ & $1.8 \pm 1.0$ & $1.9 \pm 1.0$ \\
\hline Push or Strain (1-5) & $1.1 \pm 0.4$ & $1.2 \pm 0.5$ & $1.2 \pm 0.6$ & $1.1 \pm 0.3$ & $1.1 \pm 0.5$ & $1.1 \pm 0.4$ & $1.2 \pm 0.5$ \\
\hline Pain or Burning (1-5) & $1.0 \pm 0.2$ & $1.1 \pm 0.4$ & $1.1 \pm 0.4$ & $1.1 \pm 0.4$ & $1.0 \pm 0.2$ & $1.1 \pm 0.3$ & $1.1 \pm 0.3$ \\
\hline Total Bladder Function Score (3-15) & $4.4 \pm 1.4$ & $4.2 \pm 1.4$ & $3.9 \pm 1.4$ & $3.8 \pm 1.2$ & $4.0 \pm 1.3$ & $4.0 \pm 1.3$ & $4.1 \pm 1.3$ \\
\hline$p$-value from preop & - & 0.2215 & 0.0667 & 0.0035 & 0.0007 & 0.0057 & 0.8889 \\
\hline Sleeve $(N)$ & 88 & 95 & 49 & 32 & 39 & 32 & 11 \\
\hline Urgent Bowel Movements (1-5) & $1.8 \pm 0.9$ & $1.7 \pm 0.9$ & $1.6 \pm 0.9$ & $1.5 \pm 0.7$ & $1.6 \pm 0.9$ & $1.5 \pm 0.8$ & $1.6 \pm 1.0$ \\
\hline Abdominal Cramping or Pain (1-5) & $1.7 \pm 0.8$ & $2.1 \pm 1.0$ & $1.6 \pm 1.0$ & $1.4 \pm 0.8$ & $1.5 \pm 0.9$ & $1.6 \pm 1.0$ & $1.5 \pm 0.8$ \\
\hline Loose or Liquid Stool (1-5) & $2.1 \pm 0.9$ & $2.5 \pm 1.3$ & $1.9 \pm 1.1$ & $1.7 \pm 0.9$ & $1.8 \pm 1.1$ & $1.8 \pm 1.2$ & $1.5 \pm 1.0$ \\
\hline Constipated (1-5) & $2.0 \pm 1.0$ & $2.4 \pm 1.3$ & $2.8 \pm 1.3$ & $2.6 \pm 1.4$ & $2.3 \pm 1.1$ & $1.9 \pm 1.1$ & $1.7 \pm 1.0$ \\
\hline Total Bowel Function Score (4-20) & $7.7 \pm 2.4$ & $8.7 \pm 2.6$ & $7.8 \pm 2.7$ & $7.3 \pm 2.4$ & $7.3 \pm 2.7$ & $6.8 \pm 2.9$ & $6.5 \pm 2.9$ \\
\hline$p$-value from preop & - & 0.3175 & 0.2066 & 0.0589 & 0.9103 & 0.4664 & 0.4618 \\
\hline Less than $2 \mathrm{~h}(1-5)$ & $2.4 \pm 1.3$ & $1.9 \pm 1.0$ & $1.6 \pm 1.0$ & $1.5 \pm 0.6$ & $1.5 \pm 0.7$ & $1.6 \pm 0.9$ & $1.6 \pm 0.9$ \\
\hline Push or Strain (1-5) & $1.1 \pm 0.4$ & $1.2 \pm 0.7$ & $1.2 \pm 0.7$ & $1.2 \pm 0.8$ & $1.0 \pm 0.2$ & $1.0 \pm 0.2$ & $1.0 \pm 0.0$ \\
\hline Pain or Burning (1-5) & $1.1 \pm 0.3$ & $1.1 \pm 0.4$ & $1.1 \pm 0.5$ & $1.1 \pm 0.2$ & $1.0 \pm 0.0$ & $1.0 \pm 0.2$ & $1.0 \pm 0.0$ \\
\hline Total Bladder Function Score (3-15) & $4.5 \pm 1.4$ & $4.2 \pm 1.5$ & $3.9 \pm 1.5$ & $3.8 \pm 1.3$ & $3.5 \pm 0.7$ & $3.7 \pm 1.0$ & $3.6 \pm 0.9$ \\
\hline$p$-value from preop & - & 0.0172 & 0.0685 & 0.1199 & 0.0261 & 0.0445 & 0.0465 \\
\hline \multicolumn{8}{|l|}{$p$-values Gastric Bypass vs. Sleeve } \\
\hline Total Bowel Function Score (4-20) & 0.3619 & 0.6498 & 0.2537 & 0.6102 & 0.8037 & 0.0314 & 0.1818 \\
\hline Total Bladder Function Score (3-15) & 0.7069 & 0.4792 & 0.7733 & 0.7198 & 0.0934 & 0.1601 & 0.2515 \\
\hline
\end{tabular}




\section{Multivariable analysis for factors associated with bowel and bladder function}

On multivariable analysis for factors associated with function (Table 4), there is no significant effect of gender or increasing age on bowel or bladder function scores. Greater total body weight loss is associated with significantly improved bowel function, with an estimated total score reduction of about 0.26 points per additional 10 pounds (lbs) of weight loss $(-0.026 \pm 0.008, p=0.002)$.

\section{Discussion}

Our results indicate that patients undergoing bariatric surgery, primarily RYGB and sleeve gastrectomy, experience an overall improvement in bowel function, including less urgent bowel movements, less diarrhea or constipation, and less abdominal pain as early as six weeks after surgery, with significant improvement from three months through two years follow-up. While there was a significant worsening of bowel function scores at two-week follow-up, this was transient. We hypothesize this may be attributable to the narcotic effects of surgery and improvement subsequently occurs with resolution of postoperative ileus, narcotic cessation, and ambulation. Similarly, patients reported an immediate improvement in bladder function at two-week followup which was sustained through one-year follow-up. This included less dysuria, straining to void, and decreased need to urinate again within two hours of last void.

Compared to individuals with normal weight, the presence of obesity is associated with deranged bowel and bladder function, including higher rates of diarrhea, fecal, and urinary incontinence [17-19]. The concern that undergoing weight loss surgery may exacerbate these symptoms may contribute to patients opting for less effective lifestyle or medical interventions for weight loss. Our results suggest that these fears are unfounded and should be addressed early with patients who are considering weight loss surgery.

Limitations in this study include low follow-up response on postoperative questionnaires with less than $50 \%$ of identified subjects responding at each postoperative time point. Additionally, we hypothesize that patients experiencing negative symptoms may be more likely to return to clinic for follow-up assessment and thus questionnaire completion, introducing an unknown degree of selection bias into our results.

\section{Conclusion}

Bariatric surgery provides sustained improvement in postoperative bowel and bladder function. Each additional pound of total body weight loss predicts improved postoperative 
Table 4 Multivariable factors associated with SOMS bowel and bladder function over time

\begin{tabular}{|c|c|c|c|c|}
\hline & \multicolumn{2}{|l|}{ Bowel function } & \multicolumn{2}{|l|}{ Bladder function } \\
\hline & Estimate (SE) & $\mathrm{p}$-value & Estimate (SE) & $\mathrm{p}$-value \\
\hline \multicolumn{5}{|l|}{ Time } \\
\hline Preop & Reference & - & Reference & - \\
\hline 2 Weeks & $1.506(0.289)$ & $<.0001$ & $-0.397(0.157)$ & 0.0127 \\
\hline 6 Weeks & $-1.678(2.210)$ & 0.4495 & $-2.362(1.199)$ & 0.0514 \\
\hline 3 Months & $1.244(0.803)$ & 0.1244 & $-0.925(0.436)$ & 0.0363 \\
\hline 6 Months & $0.839(0.622)$ & 0.1802 & $-0.151(0.338)$ & 0.6548 \\
\hline 1 Year & $1.095(0.677)$ & 0.1089 & $-0.185(0.338)$ & 0.6548 \\
\hline 2 Years & $1.392(0.773)$ & 0.0745 & $0.416(0.420)$ & 0.6080 \\
\hline Female vs. Male & $0.534(0.337)$ & 0.1162 & $0.059(0.184)$ & 0.7512 \\
\hline Age, per year increase & $0.007(0.012)$ & 0.5266 & $0.010(0.006)$ & 0.1374 \\
\hline TBWL, per additional lb loss & $-0.026(0.008)$ & 0.0019 & $-0.004(0.004)$ & 0.3810 \\
\hline
\end{tabular}

$T B W L$ total body weight loss bowel function. These expectations should be communicated in preoperative discussions with patients considering weight loss surgery.

Funding Drs Attaar and Haggerty receive grant funding from The Intuitive Foundation [EH20-298]. Drs Linn, Haggerty, and Ujiki receive payment for lectures from Gore. Dr Haggerty is a paid consultant for Medtronic. Dr Ujiki is a board member for Boston Scientific, a paid consultant for Olympus and Cook, and receives grant funding [ERP2020 1228] and payment for lectures from Medtronic.

\section{Declarations}

Disclosures Drs Campbell, Denham, Wong, Wu, and Butt have no conflicts of interest or financial ties to disclose. Eliza Conaty and Kristine Kuchta have no conflicts of interest or financial ties to disclose.

\section{References}

1. Flegal KM, Kruszon-Moran D, Carroll MD et al (2016) Trends in obesity among adults in the United States, 2005 to 2014. JAMA 315:2284-2291. https://doi.org/10.1001/jama.2016.6458

2. Campos GM, Khoraki J, Browning MG et al (2020) Changes in utilization of bariatric surgery in the United States from 1993 to 2016. Ann Surg 271:201-209. https://doi.org/10.1097/SLA.00000 00000003554

3. Schauer PR, Bhatt DL, Kirwan JP et al (2017) Bariatric surgery versus intensive medical therapy for diabetes-5-year outcomes. $\mathrm{N}$ Engl J Med 376:641-651. https://doi.org/10.1056/NEJMoa1600 869

4. Sudan R, Jain-Spangler K (2018) Tailoring bariatric surgery: sleeve gastrectomy, Roux-en-Y Gastric bypass and biliopancreatic diversion with duodenal switch. J Laparoendosc Adv Surg Tech A 28:956-961. https://doi.org/10.1089/lap.2018.0397

5. Aftab H, Risstad H, Søvik TT et al (2014) Five-year outcome after gastric bypass for morbid obesity in a Norwegian cohort. Surg Obes Relat Dis 10:71-78. https://doi.org/10.1016/j.soard.2013. 05.003
6. Buchwald H, Avidor Y, Braunwald E et al (2004) Bariatric surgery: a systematic review and meta-analysis. JAMA 292:17241737. https://doi.org/10.1001/jama.292.14.1724

7. Potoczna N, Harfmann S, Steffen R et al (2008) Bowel habits after bariatric surgery. Obes Surg 18:1287-1296. https://doi.org/ 10.1007/s11695-008-9456-4

8. Søvik TT, Karlsson J, Aasheim ET et al (2013) Gastrointestinal function and eating behavior after gastric bypass and duodenal switch. Surg Obes Relat Dis 9:641-647. https://doi.org/10.1016/j. soard.2012.06.006

9. Boerlage TCC, Westerink F, van de Laar AWJM et al (2019) Gastrointestinal symptoms before and after laparoscopic Roux-en-Y gastric bypass: a longitudinal assessment. Surg Obes Relat Dis 15:871-877. https://doi.org/10.1016/j.soard.2019.03.018

10. El Labban S, Safadi B, Olabi A (2015) The effect of Roux-en$\mathrm{Y}$ gastric bypass and sleeve gastrectomy surgery on dietary intake, food preferences, and gastrointestinal symptoms in postsurgical morbidly obese Lebanese subjects: a cross-sectional pilot study. Obes Surg 25:2393-2399. https://doi.org/10.1007/ s11695-015-1713-8

11. Petereit R, Jonaitis L, Kupčinskas L et al (2014) Gastrointestinal symptoms and eating behavior among morbidly obese patients undergoing Roux-en-Y gastric bypass. Medicina (Kaunas) 50:118-123. https://doi.org/10.1016/j.medici.2014.06.009

12. Clements RH, Gonzalez QH, Foster A et al (2003) Gastrointestinal symptoms are more intense in morbidly obese patients and are improved with laparoscopic Roux-en-Y gastric bypass. Obes Surg 13:610-614. https://doi.org/10.1381/096089203322190835

13. Foster A, Laws HL, Gonzalez QH et al (2003) Gastrointestinal symptomatic outcome after laparoscopic Roux-en-Y gastric bypass. J Gastrointest Surg 7:750-753. https://doi.org/10.1016/ s1091-255x(03)00092-1

14. Ballem N, Yellumahanthi K, Wolfe M et al (2009) Gastrointestinal symptom improvement after Roux-en-Y gastric bypass: longterm analysis. Surg Obes Relat Dis 5:553-558. https://doi.org/10. 1016/j.soard.2008.11.013

15. Arumugaswamy PR, Singla V, Singh $P$ et al (2021) Impact of bariatric surgery on urinary incontinence in morbidly obese individuals. Surg Endosc. https://doi.org/10.1007/s00464-021-08818-y

16. Mechanick JI, Youdim A, Jones DB et al (2013) Clinical practice guidelines for the perioperative nutritional, metabolic, and nonsurgical support of the bariatric surgery patient-2013 update: cosponsored by American Association of Clinical Endocrinologists, The Obesity Society, and American Society for Metabolic 
\& Bariatric Surgery. Obesity (Silver Spring) 21(Suppl 1):S1-27. https://doi.org/10.1002/oby.20461

17. Poylin V, Serrot FJ, Madoff RD et al (2011) Obesity and bariatric surgery: a systematic review of associations with defecatory dysfunction. Colorectal Dis 13:e92-103. https://doi.org/10.1111/j. 1463-1318.2011.02584.x

18. Mohamed F, Jeram M, Coomarasamy C et al (2021) Does bariatric surgery improve faecal incontinence? A systematic review and meta-analysis. Obes Surg 31:2942-2953. https://doi.org/10.1007/ s11695-021-05360-7
19. Roberson EN, Gould JC, Wald A (2010) Urinary and fecal incontinence after bariatric surgery. Dig Dis Sci 55:2606-2613. https:// doi.org/10.1007/s10620-010-1190-9

Publisher's Note Springer Nature remains neutral with regard to jurisdictional claims in published maps and institutional affiliations. 